\title{
A study of solar and interplanetary parameters of CMEs causing major geomagnetic storms during SC 23
}

\author{
C. Oprea ${ }^{1}$, M. Mierla ${ }^{1,2,3}$, D. Beşliu-Ionescu ${ }^{1,4}$, O. Stere ${ }^{1}$, and G. Mariş Muntean ${ }^{1}$ \\ ${ }^{1}$ Institute of Geodynamics of the Romanian Academy, 19-21 Jean-Louis Calderon, Bucharest, Romania \\ ${ }^{2}$ Research Center for Atomic Physics and Astrophysics, Faculty of Physics, University of Bucharest, Romania \\ ${ }^{3}$ Royal Observatory of Belgium, Brussels, Belgium \\ ${ }^{4}$ MoCA, School of Mathematical Sciences, Monash University, Clayton, Victoria 3800, Australia
}

Correspondence to: C. Oprea (const_oprea@yahoo.com)

Received: 5 September 2012 - Revised: 26 June 2013 - Accepted: 28 June 2013 - Published: 1 August 2013

\begin{abstract}
In this paper we analyse 25 Earth-directed and strongly geoeffective interplanetary coronal mass ejections (ICMEs) which occurred during solar cycle 23, using data provided by instruments on SOHO (Solar and Heliospheric Observatory), ACE (Advanced Composition Explorer) and geomagnetic stations. We also examine the in situ parameters, the energy transfer into magnetosphere, and the geomagnetic indexes. We compare observed travel times with those calculated by observed speeds projected into the plane of the sky and de-projected by a simple model. The best fit was found with the projected speeds. No correlation was found between the importance of a flare and the geomagnetic Dst (disturbance storm time) index. By comparing the in situ parameters with the Dst index we find a strong connection between some of these parameters (such as Bz, Bs $\cdot V$ and the energy transfer into the magnetosphere) with the strength of the geomagnetic storm. No correlation was found with proton density and plasma temperature. A superposed epoch analysis revealed a strong dependence of the Dst index on the southward component of interplanetary magnetic field, Bz, and to the Akasofu coupling function, which evaluates the energy transfer between the ICME and the magnetosphere. The analysis also showed that the geomagnetic field at higher latitudes is disturbed before the field around the Earth's equator.
\end{abstract}

Keywords. Magnetospheric Physics (Solar windmagnetosphere interactions; Storms and substorms) Solar Physics, Astrophysics, and Astronomy (Flares and mass ejections)

\section{Introduction}

Coronal mass ejections (CMEs) are huge quantities of solar magnetised plasma released into interplanetary (IP) space (see, e.g. the review by Hudson et al., 2006; Webb and Howard, 2012). CMEs that are detected in situ by space missions are called interplanetary coronal mass ejections (ICMEs) (Zurbuchen and Richardson, 2006; Jian et al., 2006). Some features observed in CMEs are not found in ICMEs and vice versa. For example, the bright core of the CME composed of cooler and denser prominence material is difficult to detect in interplanetary space. However, for isolated cases this prominence plasma (supposedly chromospheric material) was observed in situ (Schwenn et al., 1980; Gosling et al., 1980). Some ICMEs show shocks in interplanetary space, but these are very difficult to detect in the coronagraph data (Rodriguez et al., 2005). Usually, high speed CMEs $\left(v>1500 \mathrm{~km} \mathrm{~s}^{-1}\right)$ are more likely to produce shocks which can be observed in white-light images (Ontiveros and Vourlidas, 2009). Generally, ICMEs are detected by a series of characteristic signatures. In a review, Zurbuchen and Richardson (2006) list these signatures as the sudden increase of speed, the increase of magnetic field magnitude, the decrease in proton temperature, the rotation of the magnetic field, the small plasma beta, etc. Very few ICMEs possess all possible signatures together and usually at least three signatures are required to identify an ICME (Jian et al., 2006).

Generally, an ICME corresponds to one CME from the Sun, but during solar maximum more than one CME can be launched from the Sun in a short interval. These CMEs can interact in interplanetary space and arrive at the spacecraft as 
one complex ICME (Gopalswamy et al., 2001; Wang et al., 2003).

In general, the CMEs reaching Earth's magnetosphere produce large perturbations of the geomagnetic field known as geomagnetic storms (Gonzalez et al., 1994). These CMEs predominantly originate from sources near the central meridian, mostly from the Western Hemisphere (Srivastava and Venkatakrishnan, 2004; Zhang et al., 2007). The most geoeffective tend to be the energetic frontside halo CMEs, which are associated with larger, soft X-ray flares (Gopalswamy et al., 2007). The variation of the Dst geomagnetic index shows a quantitative measurement of the geomagnetic perturbation and can be correlated with some solar parameters. The first indication of a geomagnetic storm is shown by a decrease of this index. Geomagnetic storms are classified according to Dst magnitude: small (Dst $<-30 \mathrm{nT}$ ), moderate $(-50 \mathrm{nT}>$ Dst $>-100 \mathrm{nT})$, and intense (Dst $<-100 \mathrm{nT})$ (Gonzalez et al., 1994). We refer to the storms with Dst $<-150 \mathrm{nT}$ as major geomagnetic storms.

During the solar cycle 23 (SC 23) (June 1996-December 2008), there were 25 major geomagnetic storms for which unique CME signatures were observed at the Sun.

The analysis of the chain CMEs - ICMEs - major geomagnetic storms is a subject that has been intensively studied in recent years (see, e.g. Huttunen et al., 2002; Srivastava and Venkatakrishnan, 2004; Zhang et al., 2007; Echer et al., 2008; Gopalswamy et al., 2005, 2007, 2008; Gopalswamy, 2008 , etc.).

The purpose of our study is to conduct a complete survey for the solar and interplanetary parameters that could have created the major geomagnetic storms (Dst $<-150 \mathrm{nT}$ ) during SC 23. The list of our events is a subset of geomagnetic storms analysed by Zhang et al. (2007), who analysed all SC 23 storms with Dst $<-100 \mathrm{nT}$. Unlike their analysis, we do not discard any CMEs that may have been associated with the same storm. Because of this, our list contains 57 possible CMEs for the 25 geomagnetic storms, while the CMEs list of Zhang et al. (2007) contains 25 CMEs producing these 25 major geomagnetic storms. Note that Zhang et al. (2007) also list multiple CMEs associated with one geomagnetic storm, but the first of these multiple CMEs is considered the principal solar driver. We apply the analysis to both lists (ours and Zhang's CME list) and compare the results. We also analyse the in situ and geomagnetic signatures through correlation coefficient analysis and superposed epoch analysis.

\section{Data description}

During solar cycle 23, there were 28 major geomagnetic storms (Dst $\leq-150 \mathrm{nT}$ ). Out of these, we found associated CMEs for 25 events (for two of them in 1998 there were no data available as SOHO was not operational, and for the one event in 2002 no clear halo CME could be found). Therefore in the present study, only 25 events which occurred during
SC 23 (1996-2008) have been reported. The first CME from our database was recorded by LASCO (Large Angle and Spectrometric Coronagraph) on May 1998 and the last one in August 2005. The likely sources of these storms were 57 halo coronal mass ejections which were directed towards Earth starting 2 to 5 days before the initiation of each storm. The large number of CMEs (57) responsible for the 25 geomagnetic storms shows that some CMEs likely interacted in interplanetary space and arrived at Earth as a complex event.

\subsection{Catalog description}

The data were gathered in a table (available as electronic material) as follows.

- The first set of columns shows the solar signatures: date and time of the CME observed by LASCO-C2; the angular width of the CME (full or partial halo); the projected speed and the projected height at which this speed was measured; the acceleration (the kinematic parameters were taken from: http://cdaw.gsfc.nasa.gov/ CME_list/); source type (flare or prominence), start time and location on the solar disk of the source; and Hale magnetic type for the active region (data taken from http://solarmonitor.org/).

- The second set of columns shows the interplanetary signatures: disturbance time; beginning- and end-time for the ICME (data taken from http://www.ssg.sr.unh.edu/ mag/ace/ACElists/ICMEtable.html); maximum speed and the time this speed was registered; average speed; average value of the interplanetary magnetic field; the minimum value of $\mathrm{Bz}$ and the time this value of $\mathrm{Bz}$ was registered; proton density; temperature (mean values) (data taken from http://omniweb.gsfc.nasa.gov/form/ $\mathrm{dx} 1 . \mathrm{html})$; and the type of the interplanetary phenomena (ICME or ejecta,magnetic cloud (MC), etc.). The disturbance time is defined as the time when the ICME shock was recorded by the spacecraft. If the shock is missing, then the disturbance time coincides with the beginning of the ejecta. In our study, ICME refers to the ejecta as defined by Rouillard (2011) (i.e. the whole interplanetary disturbance excluding the shock, the sheath $(\mathrm{SH})$ and the compression region).

- The third set of columns shows the geomagnetic signatures: minimum value of Dst, and date and time the minimum value of Dst was registered (data taken from http://omniweb.gsfc.nasa.gov/form/dx1.html).

\subsection{Solar signatures}

Most of the 57 CMEs which we studied have their origins in complex active regions ( $\beta \gamma$ or $\beta \gamma \delta$ magnetic configuration). They were all halo CMEs, either full (angular width around the occulter of 360 degrees) or partial (angular width around the occulter between 120 and 360 degrees). The associated 
flares range from C2.0 to X17.2 class flares. Six events out of 57 have no flare association. There were 15 C-class, 25 M-class and $11 \mathrm{X}$-class flares. A total 14 events were associated with erupting or disappearing filaments. All the events, except 2 (from 20 September 1999 and 6 September 2002), had the source region associated with a NOAA active region (AR).

\subsection{In situ signatures}

In this study, we adopt the definition of an ICME as given in Zurbuchen and Richardson (2006). In general, the entire solar wind region altered by a solar transient includes the shock, the sheath, solar wind pile-up or compression region, "driver" or ejecta or ICME, plus ejecta wake (the trailing edge of the ICME) or CME legs (Rouillard, 2011). Magnetic clouds are a subclass of ICMEs characterized by smooth rotation of the magnetic field, low plasma beta and low temperature (Burlaga et al., 1982; Forsyth et al., 2001). The disturbances observed in situ are summarized in Table 1. Of the 25 events studied here, one was an ICME (without an MC signature), one was an MC, six had SHs and ICMEs, ten had SHs and MCs, and seven were complex events.

Our data list includes the shock (if associated) and the beginning- and end-time of the ejecta. The mean values of parameters (temperature, density, speeds, etc.) are calculated for the period of the ejecta only.

\subsection{Geomagnetic signatures}

The geomagnetic storms produced by the CMEs included in our list are represented by the minimum value of Dst index during the corresponding geomagnetic storms. The minimum Dst index varies from $-422 \mathrm{nT}$ to $-159 \mathrm{nT}$. Most of the storms started with a sudden commencement (SSC); only two of them showed a gradual commencement (i.e. on 6 April 2000 and 3 October 2001; source: http: //www.spacescience.ro/new1/GS_HSS_Catalogue.htm). The storm of 28 October 2001 had a SSC registered at 13:16 (source: ftp://ftp.ngdc.noaa.gov/STP/SOLAR_DATA/ SUDDEN_COMMENCEMENTS/STORM2.SSC), and the Dst minimum value registered later at 11:00. This SSC could have been caused by the ICME inhomogenous signatures, classified as a complex event of two CMEs (of 24 and 25 October 2001). The storm on 30 October 2003, 22:00 UTC, has an uncertain commencement due to overlap with a previous disturbance.

\section{Data analysis}

This section is divided into five steps. In the first two steps we analyse the connection between the solar signatures (Xray flare importance, CME speed, etc.) and the strength of the geomagnetic storms. We compared our results with the corresponding results of Zhang et al. (2007) (referred to as
Zhang CME list in this manuscript). Those authors took the most probable CME that may have caused the corresponding ICME signatures, while in the present study we considered all CMEs. A comparison between the two studies is made throughout this paper. In the third step we analyse the geomagnetic storms, depending on different phases of the solar cycle 23. Different phenomena associated with in situ signatures are also compared with the phases of the SC 23. In the fourth step, described in the following part, we calculate the correlation coefficients between different ICME physical quantities (speed, temperature, density, etc.) and Dst value. Another subsection is dedicated to describing the fifth and final step: the superposed epoch analysis for interplanetary structures parameters and geomagnetic indexes (Dst and $\mathrm{Kp}$ ).

\subsection{CME-flare dependence}

As most of the literature states, there is not a one-to-one correspondence between CMEs and flares. The general opinion is that both flares and CMEs are different manifestations of a magnetic field reorganisation (Harrison, 1995). One of the purposes of this study is to see if there is any relationship between the strength of the geomagnetic storm and the flare importance factor, $Q_{x}$, which evaluates the energy emitted by the flare in the range 1-8 $\AA$.

The 25 ICMEs in our study are correlated with 57 eruptive events, out of which 50 are associated with X-ray flares. Following the work of Maris et al. (2002), we computed the importance factor of these flares $\left(Q_{x}\right)$ in order to correlate it with the strength of the resulting geomagnetic storm. $Q_{x}$ is defined as

$Q_{x}=i_{x} \cdot t_{x}$,

where $i_{x}$ is the intensity scale of the importance of X-ray flare spectral class and $t_{x}$ is the duration of the flare in minutes. The duration of a flare is the time difference between the beginning and end of the flare, as taken from the GOES flare list (ftp://ftp.ngdc.noaa.gov/STP/space-weather/ solar-data/solar-features/solar-flares/x-rays/goes/).

We plotted $Q_{x}$ as function of Dst in Fig. 1. We see that there is no correlation between the flare importance factor and the Dst index. It can be observed that, in general, more than one flare was associated with the same geomagnetic storm. Nevertheless, each of the two CMEs associated with the strongest flares, class X10 (29 October 2003) and class X17.2 (28 October 2003), produced severe geomagnetic storms, i.e. Dst of $-383 \mathrm{nT}$ and $-353 \mathrm{nT}$, respectively. The importance of the X17.2 flare was larger than 7000 and is not plotted in Fig. 1. The correlation coefficient between Dst and $Q_{x}$ calculated using the Zhang et al. (2007) CME list was also very small (0.11). 
Table 1. Interplanetary phenomena causing strong geomagnetic storms. The table has been made according to the phenomena described in Zhang et al. (2007).

\begin{tabular}{|c|c|c|}
\hline Abbrev. & Name & Short description \\
\hline $\mathrm{MC}$ & Magnetic cloud & $\begin{array}{l}\text { Extensions of magnetic flux ropes into interplanetary space with strong magnetic fields, } \\
\text { smooth north-south, south-north rotations and low plasma beta }\end{array}$ \\
\hline ICME & Interplanetary Coronal Mass Ejection & Ejecta without magnetic cloud structure \\
\hline SH+ICME & Sheath region and ICME & Sheath region followed by an ICME \\
\hline $\mathrm{SH}+\mathrm{MC}$ & Sheath region and $\mathrm{MC}$ & Sheath region followed by a MC \\
\hline CIR & Corotating interaction region & Interaction regions between high and low speed solar wind streams \\
\hline Complex & Complex structure & $\begin{array}{l}\text { Complex phenomena deriving from CIR and ICME or ICME and ICME interactions } \\
\text { or a shock propagating through a preceding ICME or MC }\end{array}$ \\
\hline
\end{tabular}

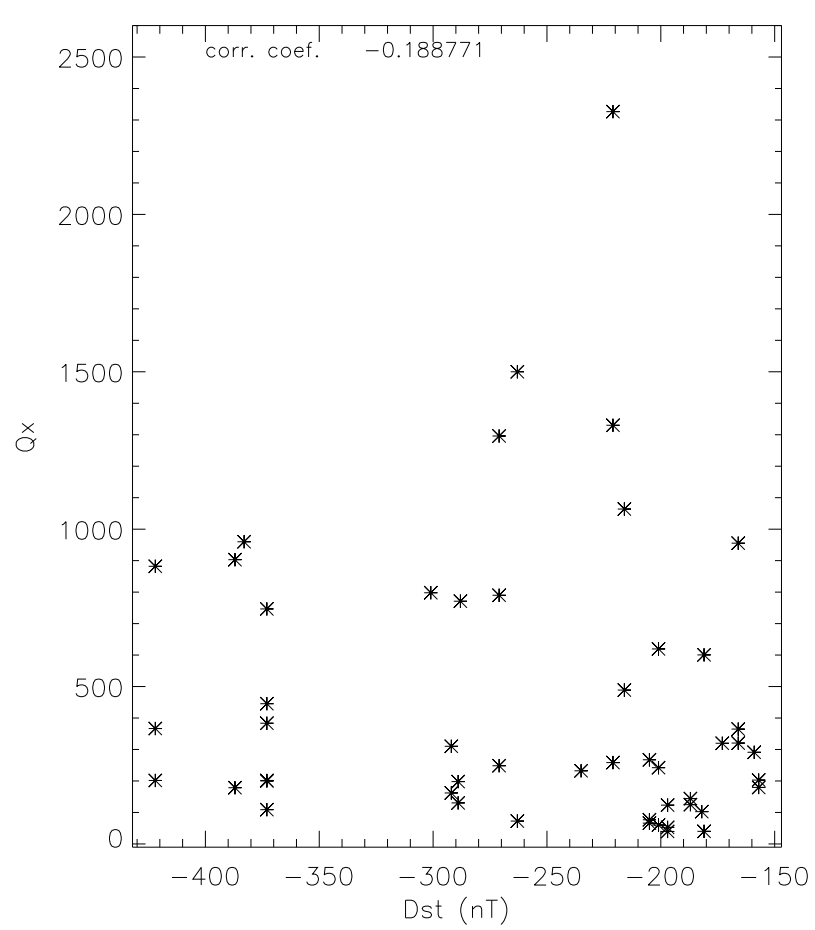

Fig. 1. The importance of soft X-ray flare indices $\left(Q_{x}\right)$ versus Dst indices. For the 28 October 2003 flare, $Q_{x}=7000$ and is not plotted in this figure in order to better show the spread of data points.

\subsection{CMEs speeds and travel times to Earth}

CMEs speeds play an important role in producing major geomagnetic storms (see, e.g. Srivastava and Venkatakrishnan, 2002). For the time period in our study (May 1998 to August 2005), the only instruments from where the CME speeds can be inferred are LASCO coronagraphs (Brueckner et al., 1995) onboard the SOHO spacecraft. This means that only one viewpoint is available, and the measured speeds (in coronagraphs field of view - FOV) are projected in the plane of the sky. These speeds are taken from the LASCO CME catalog (http://cdaw.gsfc.nasa.gov/CME_list/) and represent the speed estimated from the last measured point in the
LASCO-C3 FOV (3.7 to 30 solar radii), which is determined by fitting a second order polynomial function to the measured height-time profiles. The CMEs included in our study are halo CMEs (angular width around the occulter from 120 to 360 degrees) with source regions on the visible part of the solar disc. Usually, the feature used for obtaining the heighttime profile is the fastest one, along the leading edge at a particular position angle, observed in LASCO images. These projected speeds represent lower limits of the true, radial speeds. There are different ways to derive the true speeds: (1) point-assumption de-projection method (it assumes that the CME is a point which propagates radially from its source region); (2) geometrical fitting method (assumes the CME has a geometrical shape: cone-like, sphere-like, flux-ropelike, etc. structure). With the first method we derive speeds higher than $10000 \mathrm{~km} \mathrm{~s}^{-1}$, which are not feasible since the fastest CMEs observed are about only $3000 \mathrm{~km} \mathrm{~s}^{-1}$. These results confirm the fact that a CME is not a point source, regardless of the presence of the shock. The second approach is widely used in getting the direction of propagation and real speeds of CMEs when only one view direction is available. It has been shown that three-part limb CMEs (composed of a bright core, dark cavity and bright leading edge) are well fitted with a flux-rope like model (Thernisien et al., 2006). For halo CMEs a good approach for determining the true speed is the so-called cone model (Zhao et al., 2002; Xie et al., 2004; Cremades and Bothmer, 2005; Michalek, 2006). In this study we used a simpler approach. We assume that the CME is a sphere which expands self-similarly and propagates radially from its source region on the disk. The method is described in detail in Srivastava et al. (2009) and Mierla et al. (2012).

We used the speed derived from the sphere model to calculate the travel time of the CME to the Earth and compared it with the actual travel time. For the speed of the CME we also used the projected speed of the CME $\left(V_{\text {proj }}\right)$ and the maximum speed of the ICME $\left(V_{\text {icme }}\right)$. We assume that the CME maintains a constant speed from the Sun to the Earth. The real travel time (or shock travel time) is calculated as the difference between the time when the ICME disturbance (shock) arrived at the spacecraft and the time when the CME was last observed in LASCO-C3 images. 


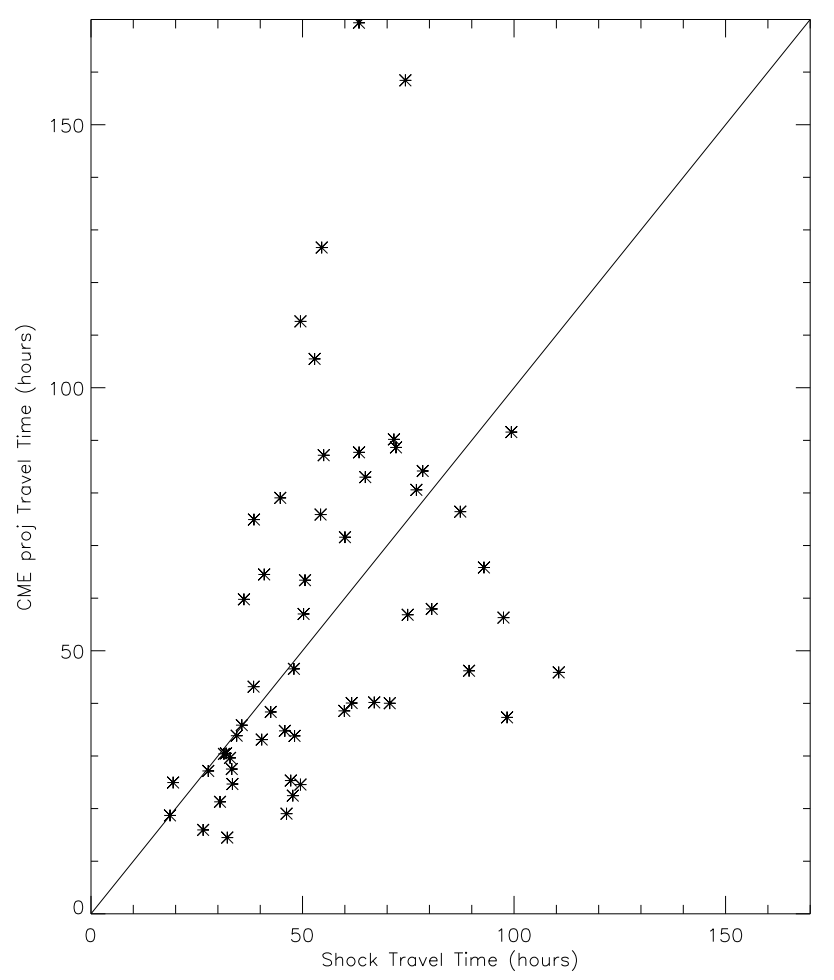

Fig. 2. Travel time calculated using the projected speeds versus real travel time.

We derived the correlation coefficient between the travel times of the CME from the Sun to Earth calculated with different speed values and the observed travel time. The best correlation with the real travel time was found for the travel time calculated using the projected speeds (correlation coefficient 0.4) (see also Fig. 2). The correlation coefficient of 0.4 is poor and does not show any significant correlation. The scatter in the plot indicates that the speed of the CME changed while propagating in interplanetary space because of the interaction with the ambient solar wind or/and interaction with other CMEs. In the case of the Zhang CME list, the best correlation to the observed travel time was found for the travel time using ICME speeds (0.8), and the travel time using projected speeds $(0.73)$.

As seen in Fig. 3, the majority of source regions of the CMEs under study lie around the central meridian. This result is in agreement with that of Srivastava and Venkatakrishnan (2004) and Zhang et al. (2007) who also found that the source regions of CMEs causing major geomagnetic storms lie close to the central meridian (latitude between -22 and 18 degrees and longitude between -30 and 72 degrees). Our study also shows that there is a slight tendency of events to originate from the western part of the solar disc.



Fig. 3. Latitude versus longitude distribution of CMEs sources.

\subsection{Dependence of in situ parameters on Dst index with solar cycle phase}

The in situ parameters of our events are analysed within event groups for different phases of the solar cycle, namely ascending, maximum and descending phase.

The SC 23 phases are as follows: the ascending (June 1997-August 1999); maximum (September 1999-July 2002) with two peaks April 2000 and November 2001, descending (August 2002-January 2006). The intervals June 1996-May 1997 and February 2006-December 2008 represent minimum phases of solar activity (Maris and Maris, 2010).

From the analysis of the geomagnetic storms presented in Table 2 and in the Catalog (the electronic material attached to the paper), it was found that in the ascending phase of SC 23 only one major geomagnetic storm (Dst $=-205 \mathrm{nT}$ ) occurred. During the maximum phase, there were 15 major geomagnetic storms $(60 \%)$, with minimum Dst values ranging between $-157 \mathrm{nT}$ and $-387 \mathrm{nT}$. In the descending phase of the solar cycle, there were nine geomagnetic storms with Dst minimum between $-181 \mathrm{nT}$ and $-422 \mathrm{nT}$. No major geomagnetic storm was observed at the minimum of solar activity.

However, if we analyse data in terms of the magnitude of the geomagnetic storms (see Table 2), we observe that in the descending phase of SC 23, four out of nine major geomagnetic storms (44\%) had a minimum Dst $<-300 \mathrm{nT}$, while in 
Table 2. The distribution of the major geomagnetic storms according to the phase of solar cycle and minimum Dst values.

\begin{tabular}{lllll}
\hline Minimum Dst (nT) & $\begin{array}{l}\text { Ascending Phase } \\
\text { (Jun 1997-Aug 1999) }\end{array}$ & $\begin{array}{l}\text { Maximum Phase } \\
\text { (Sep 1999-Jul 2002) }\end{array}$ & $\begin{array}{l}\text { Descending Phase } \\
\text { (Aug 2002-Jan 2006) }\end{array}$ & $\begin{array}{l}\text { Minimum Phase } \\
\text { (Feb 2006-Dec 2008) }\end{array}$ \\
\hline$-150 \geq$ Dst $\min >-200$ & 0 & 6 & 2 & 0 \\
$-200 \geq$ Dst $\min >-300$ & 1 & 7 & 3 & 0 \\
$-300 \geq$ Dst $\min >-450$ & 0 & 2 & 4 & 0 \\
\hline Total & 1 & 15 & 9 & 0 \\
\hline
\end{tabular}

Table 3. Geoeffectiveness of the interplanetary phenomena on the main three phases of the SC 23. Each phase is further sub-divided, depending on the strength of the geomagnetic storm: (1) $-150 \mathrm{nT} \geq$ Dst $\min >-200 \mathrm{nT}$; (2) $-200 \mathrm{nT} \geq$ Dst $\min >-300 \mathrm{nT}$; (3) $-300 \mathrm{nT} \geq \mathrm{Dst}$ $\min >-450 \mathrm{nT}$.

\begin{tabular}{|c|c|c|c|c|c|c|c|c|c|c|}
\hline \multirow[b]{2}{*}{ Type } & \multicolumn{3}{|c|}{ Ascending Phase } & \multicolumn{3}{|c|}{ Maximum Phase } & \multicolumn{3}{|c|}{ Descending Phase } & \multirow[b]{2}{*}{ Tota } \\
\hline & (1) & (2) & (3) & (1) & (2) & (3) & (1) & (2) & (3) & \\
\hline $\mathrm{MC}$ & - & - & - & 1 & - & - & - & - & - & 1 \\
\hline ICME & - & - & - & 1 & - & - & - & - & - & 1 \\
\hline $\mathrm{SH}+\mathrm{ICME}$ & - & - & - & 1 & 3 & 1 & - & 1 & - & 6 \\
\hline $\mathrm{SH}+\mathrm{MC}$ & - & - & - & 1 & 2 & 1 & 1 & 1 & 4 & 10 \\
\hline CIR & - & - & - & - & - & - & - & - & - & 0 \\
\hline Complex & - & 1 & - & 2 & 2 & - & 1 & 1 & - & 7 \\
\hline
\end{tabular}

the maximum phase of the solar cycle, two out of 15 geomagnetic storms had an minimum Dst $<-300 \mathrm{nT}$, (13\%).

In general, more than one CME is associated with a major geomagnetic storm, meaning that some CMEs interact in interplanetary space and produce a complex event. But in the case of three severe storms (Dst $<-300 \mathrm{nT}$ ) (one in July 2000 and two in October 2003), each storm corresponds to one CME. As most of the severe storms (Dst $<-300 \mathrm{nT}$ ) were observed during the descending phase of SC 23 (four compared with two at maximum of activity), we also examined if the magnitude of the storm is correlated with the magnetic polarity reversal period. According to Bilenko (2002), the north polar reversal took place in February 2001, or in May 2001 as reported by Durrant and Wilson (2003). For the Southern Hemisphere, the polar reversal took place in September 2001 (Durrant and Wilson, 2003), or in January 2002 as reported by Bilenko (2002). Thus, the poles reversed completely during the maximum of solar activity, before the descending phase started (August 2002) and when the severe storms occurred.

We statistically evaluated various types of disturbances responsible for triggering major geomagnetic storms, depending on the phases of the SC 23 activity. The relationship between the interplanetary phenomena (disturbances) and the geoeffectiveness of the storms are presented in Table 3. The analysis of the geomagnetic storms was done by dividing the Dst index value into three groups: (1) $-150 \mathrm{nT} \geq \mathrm{Dst}$ $\min >-200 \mathrm{nT}$; (2) $-200 \mathrm{nT} \geq$ Dst $\min >-300 \mathrm{nT}$; and (3) $-300 \mathrm{nT} \geq$ Dst $\min >-450 \mathrm{nT}$.
Considering the ejecta signatures types in the interplanetary space, these manifestations can be divided into several classes: MC, ICME, SH+ICME, SH+MC, CIR, and Complex (Zhang et al., 2007), as described in Table 1. According to these signatures, we observed that in the ascending phase of SC 23 only one major geomagnetic storm occurred associated with Complex structures. In the maximum phase, $15 \mathrm{ma}-$ jor geomagnetic storms occurred that were produced by MC, ICME, SH+ICME, SH+MC and Complex structures. Finally, in the descending phase, the interplanetary phenomena that caused the nine major geomagnetic storms were SH+ICME, $\mathrm{SH}+\mathrm{MC}$ and Complex structures.

Most of the storms (ten, $40 \%$ ) were produced by $\mathrm{SH}+\mathrm{MC}$, seven storms $(28 \%)$ by Complex events, six $(24 \%)$ by $\mathrm{SH}+\mathrm{ICME}$, one storm (4\%) by an MC and one storm (4\%) by an ICME alone.

In conclusion, the statistical study presented above showed that the most geoeffective phenomena are $\mathrm{SH}+\mathrm{MC}$, followed by Complex structures and then SH+ICME.

\subsection{Correlation between the IP parameters and the geomagnetic indices}

In this section we study the correlation coefficients and their significance ( $p$ value) between the Dst index and the ICME parameters. The correlation coefficient indicates the strength of a relationship, while the significance of a correlation computes the probability values of the relationship, testing the hypothesis of no correlation versus a non-zero correlation. The smaller the $p$ value is, the more significant the relation 
between parameters. The threshold for a correlation to be significant is 0.05 .

We analysed 22 of our 25 ICMEs; three were not included for analysis due to a lack of in situ data (one on 6 November 2001 and two on 30 October 2003). We computed the correlation coefficients and significance for the most important parameters of these interplanetary structures (the average values of the interplanetary magnetic field $(B)$, plasma speed $(V)$, proton density and plasma temperature) and the corresponding value of minimum Dst, as shown in Table 4.

We also calculated the correlation coefficients and significance between minimum Dst and the $\mathrm{Bz}$ component of the $\mathrm{IMF}$, the plasma speed, $\mathrm{Bs} \cdot V$ (where $\mathrm{Bs}=|\mathrm{Bz}|$ when $\mathrm{Bz}<0$ and $\mathrm{Bs}=0$ when $\mathrm{Bz} \geq 0$ ), the proton density and the plasma temperature, parameters taken at time $\left(t_{0}\right)$, one hour earlier $\left(t_{-1}\right)$, two hours earlier $\left(t_{-2}\right)$ and three hours earlier $\left(t_{-3}\right)$ than the time of minimum Dst. Another parameter correlated with minimum Dst was the total energy injected into the magnetosphere during the principal phase of the geomagnetic storm $\left(W_{\varepsilon}\right)$, computed through the Akasofu coupling function, $\varepsilon$ (see Akasofu, 1983). The Akasofu coupling function takes into consideration the processes of reconnection within the magnetosphere, as it is considered to be the principal source of the injected energy (De Lucas et al., 2007):

$\varepsilon=10^{7} V B^{2} l_{0}^{2} \sin ^{4}(\theta / 2),\left[\mathrm{J} \mathrm{s}^{-1}\right]$

where $V$ is the plasma speed, $B$ is the interplanetary magnetic field (IMF), $\theta$ is the IMF clock angle in the plane perpendicular to the Sun-Earth line, $l_{0}$ is the magnetopause radius $\left(l_{0}=7 R_{E}\right), \theta=\tan ^{-1}\left(B_{y} / B_{z}\right)$.

$$
W_{\varepsilon}=\int_{t_{\mathrm{b}}}^{t_{\mathrm{m}}} \varepsilon \mathrm{d} t,[\mathrm{~J}]
$$

where $W_{\varepsilon}$ is obtained by integrating $\varepsilon$ over the principal phase of each geomagnetic storm, from the beginning of the storm $\left(t_{\mathrm{b}}\right)$ to the time when the minimum Dst was registered $\left(t_{\mathrm{m}}\right)$. All units are in international system (SI) units of measurement.

The best correlation coefficients and significance with minimum Dst were found for $\mathrm{Bz}$ registered two and three hours earlier than minimum Dst (correlation coefficient $(r)$ of 0.76 and, 0.68 respectively; significance of $3.3 \times 10^{-5}$ and $\left.5.1 \times 10^{-4}\right)$ respectively, for Bs $\cdot V(r=-0.74$; significance $\left.=8.8 \times 10^{-5}\right)$ and for the average value of $B(r=-0.66$; significance of $9 \times 10^{-4}$ ). Also, for the total energy injected into the magnetosphere parameter, $W_{\varepsilon}$ had a good correlation with minimum Dst $\left(r=-0.71\right.$, significance $\left.=2.1 \times 10^{-4}\right)$. No significant correlation was found for the plasma temperature and proton density with the strength of the geomagnetic storm.

In Fig. 4 we graphically represent Dst versus $\mathrm{Bz}$ (top panel) and Dst versus Bs $\cdot V$ (middle panel). The IP parameters $(\mathrm{Bz}$ and $V)$ are measured two hours before minimum
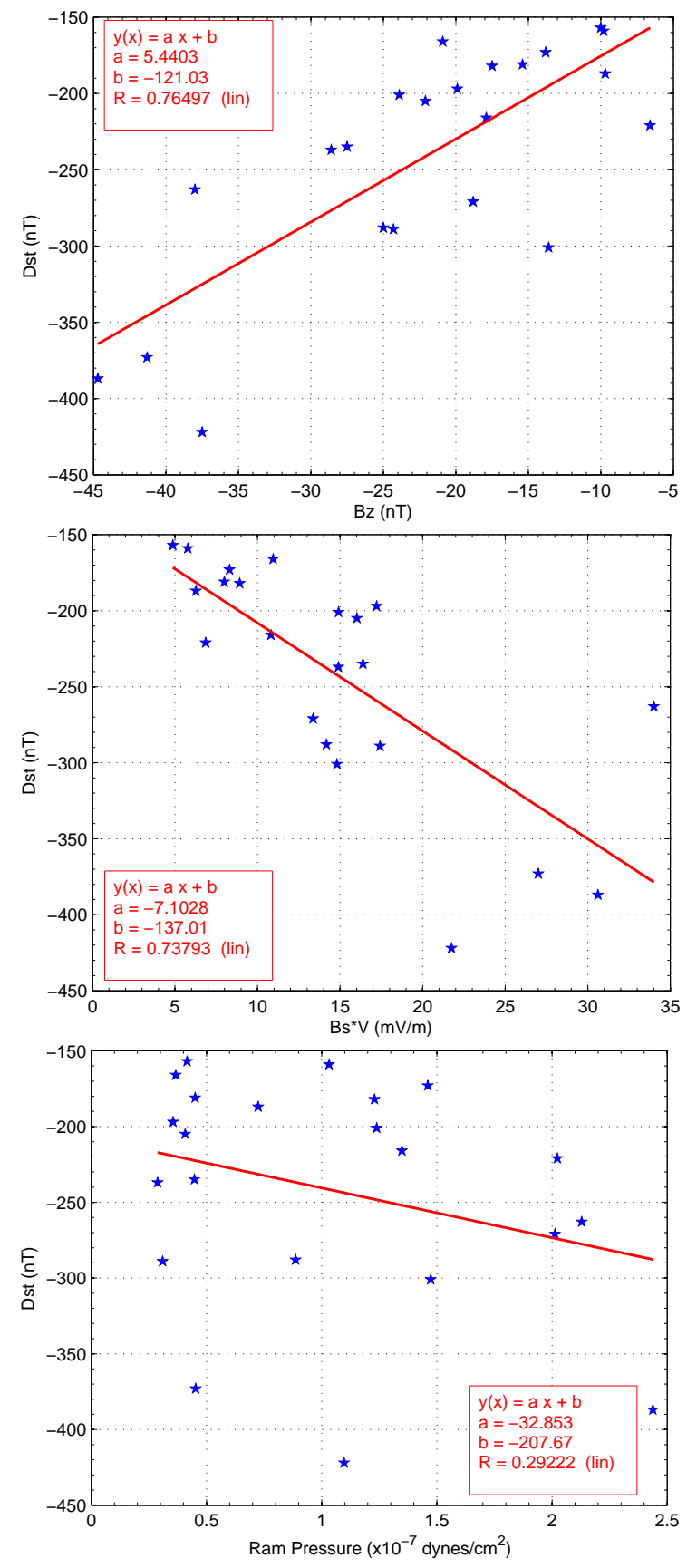

Fig. 4. Graphic representation of Dst versus various ICME parameters: Bz vs. Dst (top panel, recorded two hours before minimum Dst), Bs · V vs. Dst (middle panel, recorded two hours before minimum Dst) and ram pressure vs. Dst (bottom panel, recorded three hours before minimum Dst). The values of Bs $\cdot V$ are multiplied by $10^{-3}$.

Dst. The trend of this dependence can be fitted with a linear function, showing a high degree of dependence between the two pairs of parameters. In the bottom panel we represented the ram pressure measured three hours before the minimum Dst versus minimum Dst. The correlation coefficient 
Table 4. The correlation and significance between the interplanetary structures parameters and the minimum value of Dst. The interplanetary parameters are calculated at the same time as the Dst minimum $\left(t_{0}\right)$, one hour $\left(t_{-1}\right)$, two $\left(t_{-2}\right)$ and three hours earlier, respectively.

\begin{tabular}{|c|c|c|c|c|c|c|}
\hline \multirow[b]{2}{*}{ No Crt. } & \multirow[b]{2}{*}{ IP parameters } & \multicolumn{5}{|c|}{ Correlation coefficient $(r)$ [Significance] } \\
\hline & & IP average & IP at $t_{0}$ & IP at $t_{-1}$ & IP at $t_{-2}$ & IP at $t_{-3}$ \\
\hline 1. & $B$ & $-0.66\left[9.3 \times 10^{-4}\right]$ & - & - & - & - \\
\hline 2. & $\mathrm{Bz}$ &  & $0.09\left[6.7 \times 10^{-1}\right]$ & $0.46\left[2.9 \times 10^{-2}\right]$ & $0.76\left[3.3 \times 10^{-5}\right]$ & $0.68\left[5.1 \times 10^{-4}\right]$ \\
\hline 3. & $V$ & $-0.37\left[9.1 \times 10^{-2}\right]$ & $-0.20\left[3.6 \times 10^{-1}\right]$ & $-0.21\left[3.5 \times 10^{-1}\right]$ & $-0.21\left[3.3 \times 10^{-1}\right]$ & $-0.29\left[1.9 \times 10^{-1}\right]$ \\
\hline 4. & $\mathrm{Bs} \cdot V$ & - & $-0.28\left[2.0 \times 10^{-1}\right]$ & $-0.49\left[2.1 \times 10^{-2}\right]$ & $-0.74\left[8.8 \times 10^{-5}\right]$ & $-0.74\left[7.3 \times 10^{-5}\right]$ \\
\hline 5. & Ram pressure & - & $-0.15\left[4.9 \times 10^{-1}\right]$ & $-0.26\left[2.3 \times 10^{-1}\right]$ & $-0.07\left[7.3 \times 10^{-1}\right]$ & $-0.29\left[1.8 \times 10^{-1}\right]$ \\
\hline 6. & Proton density & $-0.25\left[2.6 \times 10^{-1}\right]$ & $-0.07\left[7.5 \times 10^{-1}\right]$ & $-0.13\left[5.7 \times 10^{-1}\right]$ & $0.07\left[7.4 \times 10^{-1}\right]$ & $-0.09\left[6.9 \times 10^{-1}\right]$ \\
\hline 7. & Plasma temperature & $-0.23\left[3.1 \times 10^{-1}\right]$ & $0.16\left[4.8 \times 10^{-1}\right]$ & $0.18\left[4.1 \times 10^{-1}\right]$ & $0.03\left[8.9 \times 10^{-1}\right]$ & $0.01\left[9.6 \times 10^{-1}\right]$ \\
\hline 8. & $W_{\varepsilon}$ & $-0.71\left[2.1 \times 10^{-4}\right]$ & - & - & - & - \\
\hline
\end{tabular}


Fig. 5. Superposed epoch analysis for the hourly mean values of Dst and Bz (upper left panel), Dst and Akasofu coupling function (lower left panel) and for the 3-hourly mean values of Dst and Kp (upper right panel), Kp and Akasofu coupling function (lower right panel).

indicates a weak dependence for this pair of parameters. This is different from the result of Srivastava and Venkatakrishnan (2004) who found a better correlation between the two quantities.

The minimum Dst versus proton density and the minimum Dst versus plasma temperature, values taken in the same manner as in the upper panels cases, do not show any correlation.

Even if the amount of data considered for this study was small (25), it is obvious that certain ICME parameters have a good correlation with the Dst index and thus the intensity of the geomagnetic storm.

\subsection{Superposed epoch analysis}

In this section we present the superposed epoch analysis (e.g. Guo et al., 2011) of different IP parameters, the Akasofu coupling function, the Kp geomagnetic index and the Dst index.

The parameters that characterize the behaviour of the IP structures and the geomagnetic response used in this analysis are the hourly mean values of $B, \mathrm{Bz}$, plasma speed, plasma temperature, proton density, Dst, Kp, and the Akasofu coupling function. In this analysis, we considered the time at which the Dst reached minimum values as $t=0$. We selected the data recorded $24 \mathrm{~h}$ before the Dst minimum to 
$48 \mathrm{~h}$ after the minimum, as this interval is sufficient to reveal the changes and the evolution of the studied parameters.

To have a better overview, we represented two parameters on each plot in Fig. 5. For this purpose, we computed the hourly mean values of Dst index (red line) and Bz (blue line), in the upper left panel and the Dst index (red line) and Akasofu coupling function (blue line), in the lower left panel. Due to the fact that the Kp index is determined on a three-hourly interval, we calculated the 3-hourly mean values of Dst and Akasofu coupling function in order to superpose these parameters onto the $\mathrm{Kp}$ index values. We represented the Dst index (red line) and Kp index (blue line) in the upper right panel, and the Kp index (red line) and Akasofu coupling function (blue line) in the lower right panel of Fig. 5. All the values are averages of the 22 events.

The superposed epoch analysis revealed the strong dependence of the geomagnetic storm intensity on the southward component of the interplanetary magnetic field, $\mathrm{Bz}$, which leads to magnetic reconnection between the IP structures and Earth's front-side magnetic field. This strong dependence between the storm intensity and $\mathrm{Bz}$ has been well established by the past studies (see, e.g. Wu and Lepping, 2002; Srivastava and Venkatakrishnan, 2004; Echer et al., 2008).

A tight dependence between the Akasofu coupling function and both the Dst and Kp values is also evident (see, e.g. Akasofu, 1983; De Lucas et al., 2007). This fact reveals the significant role played by the reconnection in the amount of the energy injected into the magnetosphere in the main phase of each geomagnetic storm.

Upper-right panel of Fig. 5 shows that Kp value increases more rapidly than the decrease of the Dst index, revealing the fact that the field at higher latitudes is disturbed before the field around the Earth's equator (see e.g. Davis et al., 1997).

\section{Summary}

The aim of this study was to survey the major geomagnetic storms that took place in SC 23. We analysed the sources, the kinematics and the interplanetary parameters of $57 \mathrm{CMEs}$ that likely caused 25 major geomagnetic storms in solar cycle 23.

All the 57 CMEs were halos and were directed towards Earth. The majority of them emerged complex active regions ( $\beta \gamma$ or $\beta \gamma \delta$ magnetic configurations).

The associated flares had a wide energy range, from C2.0 to X17.2. No correlation was found between the geoeffectiveness and the importance of a flare.

The CME speed analysis revealed that the projected speeds gave a better estimate of the observed travel time than the ones calculated from a self-similarly expending sphere model. The possible explanations are that the model used to derive the true speed is too simple and/or the CME propagates with the same speed in all directions. The CMEs in this study show both acceleration and deceleration while propagating in interplanetary space due to the interaction with the ambient solar wind or/and the interaction with other CMEs.

The distribution of the major geomagnetic storms over different phases of SC 23 showed that there were no major storms during the cycle minimum, only one storm with clear solar signatures in the ascending phase, fifteen during the maximum, and nine in the descending phase.

Overall, ten storms $(40 \%)$ were produced by $\mathrm{SH}+\mathrm{MC}$, seven storms $(28 \%)$ by Complex structures, six $(24 \%)$ by $\mathrm{SH}+\mathrm{ICME}$, one storm (4\%) by an MC and one storm (4\%) by an ICME.

The best correlation coefficients and significance were found between minimum value of Dst and Bz measured two and three hours earlier than minimum Dst. A good correlation was also found between the total energy injected into the magnetosphere and Dst. No significant correlation was found between the solar wind plasma temperature, proton density and the strength of the geomagnetic storm.

The superposed epoch analysis revealed the strong dependence of the geomagnetic storm intensity to the southward component of the interplanetary magnetic field, $\mathrm{Bz}$, and to the Akasofu coupling function, demonstrating the significant role played by the reconnection between interplanetary and geomagnetic fields and thus the amount of the energy injected into the magnetosphere in the main phase of each geomagnetic storm (see also, Gonzalez and Echer, 2005; Zhang et al., 2007; Echer et al., 2008). The analysis also showed that the field at higher latitudes is disturbed earlier than the field around the Earth equator.

\section{Supplementary material related to this article is available online at: http://www.ann-geophys.net/31/1285/ 2013/angeo-31-1285-2013-supplement.pdf.}

Acknowledgements. This research was supported from the CNCSIS project TE_2, No. 73/11.08.2010. We acknowledge the use of SOHO, ACE and geomagnetic data. We thank the anonymous referees for their useful comments, which greatly improved the paper.

Topical Editor M. Pinnock thanks N. Srivastava and one anonymous referee for their help in evaluating this paper.

\section{References}

Akasofu, S.-I.: Solar-wind disturbances and the solar-wind magnetosphere energy coupling function, Space Sci. Rev., 34, 173-183, 1983.

Bilenko, I. A.: Coronal holes and the solar polar field reversal, Astron. Astrophys., 396, 657-666, 2002.

Brueckner, G. E., Howard, R. A., Koomen, M. J., Korendyke, C. M., Michels, D. J., Moses, J. D., Socker, D. G., Dere, K. P., Lamy, P. L., Llebaria, A., Bout, M. V., Schwenn, R., Simnett, G. M., Bedford, D. K., and Eyles, C. J.: The Large Angle Spectroscopic Coronagraph (LASCO), Solar Phys., 162, 357-625, 1995. 
Burlaga, L. F., Klein, L., Sheeley Jr., N. R., Michels, D. J., Howard, R. A., Koomen, M. J., Schwenn, R., and Rosenbauer, H.: A magnetic cloud and a coronal mass ejection, Geophys. Res. Lett., 9, 1317-1320, 1982.

Cremades, H. and Bothmer, V.: Geometrical Properties of Coronal Mass Ejections, IAU Symposium Proceedings of the International Astronomical Union 226, Held 13-17 September, Beijing, edited by: Dere, K., Wang, J., and Yanm, Y., Cambridge: Cambridge University Press, 48-54, 2005.

Davis, C. J., Wild, M. N., Lockwood, M., and Tulunay, Y. K.: Ionospheric and geomagnetic responses to changes in IMF BZ: a superposed epoch study, Ann. Geophys., 15, 217-230, doi:10.1007/s00585-997-0217-9, 1997.

De Lucas, A., Gozales, W. D., Echer, E., Guarnieri, F. L., Dal Lago, A., da Silva, M. R., Vieira, L. E. A., and Schuch, N. J.: Energy balance during intense and super-intense magnetic storms using an Akasofu $\varepsilon$ parameter corrected by the solar wind dynamic pressure, J. Atmos. Sol.-Terr. Phy., 69, 1851-1863, 2007.

Durrant, C. J. and Wilson, P. R.: Observations and Simulations of the Polar Field Reversals in Cycle 23, Solar Phys., 214, 23-39, 2003.

Echer, E., Gonzalez, W. D., Tsurutani, B. T., and Gonzalez, A. L. C.:Interplanetary conditions causing intense geomagnetic storms (Dst $\leq 100 \mathrm{nT}$ ) during solar cycle 23 (1996-2006), J. Geophys. Res., 113, A05221, doi:10.1029/2007JA012744, 2008.

Forsyth, R. J., Rees, A., Balogh, A., and Smith, E. J.: Magnetic Field Observations of Transient Events at Ulysses, 1996-2000, Space Sci. Rev., 97, 217-220, 2001

Gonzalez, W. D. and Echer, E.: A study on the peak Dst and peak negative $\mathrm{Bz}$ relationship during intense geomagnetic storms, Geophys. Res. Lett., 32, L18103, doi:10.1029/2005GL023486, 2005.

Gonzalez, W. D., Joselyn, J. A., Kamide, Y., Kroehl, H. W., Rostoker, G., Tsurutani, B. T., and Vasyliunas, V. M.: What is a geomagnetic storm?, J. Geophys. Res., 99, 5771-5792, 1994.

Gopalswamy, N.: Solar connections of geoeffective magnetic structures, J. Atmos. Sol.-Terr. Phy., 70, 2078-2100, 2008.

Gopalswamy, N., Yashiro, S., Kaiser, M. L., Howard, R. A., and Bougeret, J.-L.: Radio Signatures of Coronal Mass Ejection Interaction: Coronal Mass Ejection Cannibalism?, Astrophys. J., 548, L91-L94, 2001.

Gopalswamy, N., Yashiro, S., Michalek, G., Xie, H., Lepping, R. P., and Howard, R. A.: Solar source of the largest geomagnetic storm of cycle 23, Geophys. Res. Lett., 32, L12S09, doi:10.1029/2004GL021639, 2005.

Gopalswamy, N., Yashiro, S., and Akiyama, S.: Geoeffectiveness of halo coronal mass ejections, J. Geophys. Res., 112, A06112, doi:10.1029/2006JA012149, 2007.

Gopalswamy, N., Akiyama, S., Yashiro, S., Michalek, G., and Lepping, R. P.: Solar sources and geospace consequences of interplanetary magnetic clouds observed during solar cycle 23, J. Atmos. Sol.-Terr. Phy., 70, 245-253, 2008.

Gosling, J. T., Asbridge, J. R., Bame, S. J., Feldman, W. C., and Zwickl, R. D.: Observations of large fluxes of $\mathrm{He} / \mathrm{+} /$ in the solar wind following an interplanetary shock, J. Geophys. Res., 85, 3431-3434, 1980.

Guo, J., Feng, X., Emery, B. A., Zhang, J., Xiang, C., Shen, F., and Song, W.: Energy transfer during intense geomagnetic storms driven by interplanetary coronal mass ejections and their sheath regions, J. Geophys. Res., 116, A05106, doi:10.1029/2011JA016490, 2011.

Harrison, R. A.: The nature of solar flares associated with coronal mass ejections, A\&A, 304, 585-591, 1995.

Hudson, H. S., Bougeret, J.-L., and Burkepile, J.: Coronal Mass Ejections: Overview of Observations, Space Sciences Series of ISSI, 21, ISBN 978-0-387-45086-5, Springer, 13-30, 2006.

Huttunen, K. E. J., Koskinen, H. E. J., Schwenn, R., and dal Lago, A.: Causes of major magnetic storms near the latest solar maximum, in: ESA SP-506, 1, Noordwijk, edited by: Wilson, A., ESA Publications Division, ISBN 92-9092- 816-6, 2002.

Jian, L., Russell, C. T., Luhmann, J. G., and Skoug, R. M.: Properties of Interplanetary Coronal Mass Ejections at One AU During 1995-2004, Solar Phys., 239, 393-436, 2006.

Maris, G. and Maris, O.: Rapid solar wind and geomagnetic variability during the ascendant phases of the 11-yr solar cycles, Solar and Stellar Variability: Impact on Earth and Planets, Proceedings of the International Astronomical Union, IAU Symposium, 264, 359-362, 2010.

Maris, G., Popescu, M. D., and Mierla, M.: The North-South Asymmetry of Soft X-Ray Solar Flares, Romanian Astronomical Journal, 12, 131-146, 2002.

Michalek, G.: An Asymmetric Cone Model for Halo Coronal Mass Ejections, Solar Phys., 237, 101-118, 2006.

Mierla, M., Besliu-Ionescu, D., Chiricuta, O., Oprea, C., and Maris, G.: Studies of coronal mass ejections that have produced major geomagnetic storms, Sun and Geosphere, 7, 13-16, 2012.

Ontiveros, V. and Vourlidas, A.: Quantitative Measurements of Coronal Mass Ejection-Driven Shocks from LASCO Observations, Astrophys. J., 693, 267-275, 2009.

Rodriguez, L., Woch, J., Krupp, N., Fränz, M., von Steiger, R., Cid, C., Forsyth, R., and Glaßmeier, K.-H.: Freezing-In Temperatures of Oxygen, Carbon and Iron in Magnetic Clouds, Proceedings of the Solar Wind 11/SOHO 16, "Connecting Sun and Heliosphere" Conference (ESA SP-592), 12-17 June 2005 Whistler, Canada, edited by: Fleck, B., Zurbuchen, T. H., and Lacoste, H., p. 759, 2005.

Rouillard, A. P.: Relating white light and in situ observations of coronal mass ejections: A review, J. Atmos. Sol.-Terr. Phy., 73, 1201-1213, 2011.

Schwenn, R., Rosenbauer, H., and Muehlhaeuser, K.-H.: Singlyionized helium in the driver gas of an interplanetary shock wave, Geophys. Res. Lett., 7, 201-204, 1980.

Srivastava, N. and Venkatakrishnan, P.: Relationship between CME Speed and Geomagnetic Storm Intensity, Geophys. Res. Lett., 29, 1287, doi:10.10292001GL013597, 2002.

Srivastava, N. and Venkatakrishnan, P.: Solar and interplanetary sources of major geomagnetic storms during 1996-2002, J. Geophys. Res., 109, A10103, doi:10.1029/2003JA010175, 2004.

Srivastava, N., Inhester, B., Mierla, M., and Podlipnik, B.: 3D Reconstruction of the Leading Edge of the 20 May 2007 Partial Halo CME, Solar Phys., 259, 213-225, 2009.

Thernisien, A. F. R., Howard, R. A., and Vourlidas, A.: Modeling of Flux Rope Coronal Mass Ejections, Astrophys. J., 652, 763-773, 2006.

Wang, Y. M., Ye, P. Z., and Wang, S.: Multiple magnetic clouds: Several examples during March-April 2001, J. Geophys. Res., 108, 1370, doi:10.1029/2003JA009850, 2003. 
Webb, D. F. and Howard, T. A.: Coronal Mass Ejections: Observations, Living Rev. Solar Phys., 9, 3, doi:10.12942/lrsp-2012-3, 2012.

Wu, C.-C. and Lepping, R. P.: Effects of magnetic clouds on the occurrence of geomagnetic storms: The first 4 years of Wind, J. Geophys. Res., 107, 1314, doi:10.1029/2001JA000161, 2002.

Xie, H., Ofman, L., and Lawrence, G.: Cone model for halo CMEs: Application to space weather forecasting, J. Geophys. Res., 109, A03109, doi:10.1029/2003JA010226, 2004.

Zhang, J., Richardson, I. G., Webb, D. F., Gopalswamy, N., Huttunen, E., Kasper, J. C., Nitta, N. V., Poomvises, W., Thompson, B. J., Wu, C.-C., Yashiro, S., and Zhukov, A. N.: Solar and interplanetary sources of major geomagnetic storms (Dst $\leq-100 \mathrm{nT}$ ) during 1996-2005, J. Geophys. Res., 112, A10102, doi:10.1029/2007JA012321, 2007.
Zhao, X. P., Plunkett, S. P., and Liu, W.: Determination of geometrical and kinematical properties of halo coronal mass ejections using the cone model, J. Geophys. Res., 107, 1223, doi:10.1029/2001JA009143, 2002.

Zurbuchen, T. H. and Richardson, I.: In-Situ Solar Wind and Field Signatures of Interplanetary Coronal Mass Ejections, Space Sci. Rev., 123, 31-43, 2006. 